\title{
Alloy Development and Optimisation Informed by an Understanding of Cold Dwell Fatigue Sensitivity
}

\author{
M.R. Bache ${ }^{1}$ and M. Thomas ${ }^{2}$ \\ ${ }^{1}$ Institute of Structural Materials, College of Engineering, Bay Campus, Swansea University, \\ Swansea, SA1 8EN, UK \\ 2 TIMET UK, PO Box 704, Witton, Birmingham, B6 7UR, UK
}

\begin{abstract}
$\underline{\text { Abstract }}$
Near $\alpha$ titanium alloys have demonstrated a sensitivity to fatigue loading when incorporating a dwell period at peak applied stress under room temperature. Historically, this was first reported for the relatively coarse grained variant IMI685 and later exemplified when characterising TIMETAL ${ }^{\circledR} 834$ (Ti-834). Various mechanical factors have been identified as key drivers for the phenomenon, including time on load and high $\mathrm{R}$ ratios. This indicates that sub-critical damage is accumulated via creep style mechanisms and has led to analogies with "cold creep" behaviour. The propensity of quasi-cleavage faceting at the initiation sites of fatigue cracks formed under dwell conditions eventually led to an understanding of stress redistribution between strong and weak grains. This mechanism can be accentuated by more extensive regions of common crystallographic grain orientation in the form of "macrozones". Through this fundamental understanding, new alloys can now be developed with the intent to circumvent dwell sensitivity.
\end{abstract}

The present paper will focus on the fatigue performance of TIMETAL $® 575$ (Ti-575), a recently developed alloy optimised for aero-engine applications. Ti-575 was designed for improved strength and fatigue performance. The alloy's susceptibility to dwell fatigue has been avoided through control of the microstructural evolution kinetics and associated thermo-mechanical process route to induce fine scaled, bimodal microstructure containing equiaxed primary grains and secondary $\alpha$ laths with inherent random grain orientations, thus minimising the formation of macrozones. This work will detail the dwell fatigue testing, interpretation of data and associated microstructural characterisation, including EBSD, and compare this analysis with similar results from more conventional alloys such as Ti-6Al-4V and Ti834.

\section{Introduction}

The phenomenon of cold dwell fatigue sensitivity can be traced back to the seminal research conducted at the then Royal Aircraft Establishment (RAE) at Farnborough in the United Kingdom. Following in-service fan disc failures occurring in RB-211 engines during the early 1970s [1], Evans and Gostelow eventually published the results of their detailed fatigue assessment in 1978, focussed upon the near $\alpha$ alloy IMI685 [2]. Originally designed as an alloy offering high temperature creep resistance for high pressure compressor applications, the same alloy had also been selected for the RB-211 fan disc. The work demonstrated that fatigue performance was adversely affected by the imposition of dwell periods at the peak of the load cycle and in selected microstructural variants of the alloy a reduction in fatigue life greater than an order of magnitude could be induced. Various features of those original studies at the RAE went on to form "standard practise" for subsequent practitioners investigating the dwell effect, not least the employment of a 2 minute hold time at peak load/stress to characterise dwell performance. A dwell fatigue assessment has since become an essential consideration for all new disc alloy development programmes.

Components and laboratory specimens alike, subjected to dwell fatigue failure, invariably reveal quasi-cleavage facets at the point of crack initiation. Although similar facets are induced in all near $\alpha$ alloys tested under different forms of loading, where an element of time dependency is included (e.g. example baseline low cycle fatigue (LCF), high cycle fatigue (HCF) and creep), they appear to be accentuated by dwell waveforms [3]. Originally described according to the Stroh model [4], empirical and plasticity modelling activities [5,6] have since described the formation of these facets via a process of stress re-distribution, forming as they do at sub-surface sites and within relatively "hard" grains in juxtaposition with "softer" grains. For the hexagonal based titanium alloys, these hard and soft grains correlate to a c-axis orientation near parallel to the tensile load axis or favourably inclined, to either resist or encourage slip on the basal plane, respectively. Time dependent planar slip then drives dislocations to pile up at the hard/soft grain boundary and induces an opening stress in the hard grain. This stress, acting in combination with the tensile applied stress, initiates and progressively opens the quasi-cleavage facet orientated near orthogonal to the tensile stress axis. It is this time dependency that promotes faceting under dwell scenarios.

It has since become apparent that the stress redistribution mechanism can also activate over a larger length scale, should the alloy microstructure contain regions with inhomogeneous mechanical properties as a result of significant micro-texture. An agglomeration of grains with identical or similar crystallographic orientation, now recognised by the terms "macro-zone" or "micro textured regions (MTR's)" may act as a single effective microstructural unit. Hard and soft regions within the material bulk can then lead to highly facetted zones comprised of multiple facets formed within individual $\alpha$ grains. An exemplar of such behaviour has been demonstrated by alloys such as Ti834 [7]. The formation of these macrozones is known to have its origins in the thermomechanical processing during billet breakdown and/or final component forming. By adapting these processes, it has been shown that the formation and evolution of macrozones can be controlled in $\alpha+\beta$ or near $\alpha$ alloys $[8,9]$.

Given this understanding, alloy development can be steered to optimise chemical composition and manipulate processing parameters to avoid the formation of macro-zones and thereby minimise dwell sensitivity. TIMETAL ${ }^{2} 575$ (Ti-575), a recently developed alloy optimised for aero-engine applications, was designed for improved strength and fatigue performance. The alloy's susceptibility to dwell fatigue has been avoided through control of the phase transformation kinetics and subsequent microstructural evolution and associated thermomechanical process route to induce fine scaled, bi-modal microstructure containing equi-axed primary grains and secondary $\alpha$ laths with inherent random grain orientations, thus minimising the formation of macro-zones. A comprehensive assessment incorporating low cycle fatigue experiments, pre and post mortem texture evaluations and associated fractography will be presented to illustrate the success of this approach, with comparisons to similar results from more conventional alloys such as Ti-6Al-4V and Ti-834. 


\section{Experimental Methods}

Materials

An ingot of Ti-575 was manufactured at Titanium Metals Corporation (TIMET) UK. A proprietary sequence of forging steps both above the

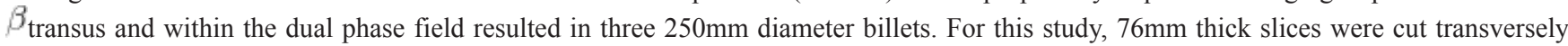
from one billet, sectioned longitudinally in $60^{\circ}$ increments from the centre. Detailed heat treatment and metallography studies were performed to identify the $\alpha$ approach curve. Based on this information, the $60^{\circ}$ "wedges" were then heated to transus $-50^{\circ} \mathrm{C}$ for 2 hours before a 3:1 forging reduction. The subsequent "pancakes", designed to mimic a typical fan disc forging, were then subjected to an "aged" heat treatment (transus $-50^{\circ} \mathrm{C} / 2 \mathrm{hr}$, Force Air Cooled + Age $500^{\circ} \mathrm{C} / 8 \mathrm{hr} \mathrm{AC}$ ), ultimately proving to be the condition to offer the strongest fatigue response whilst resisting dwell sensitivity. A relatively fine scaled bi-modal microstructure was the result, Figure 1, containing elongated primary $\alpha$ grains within a transformed $\alpha+\beta$ matrix. An electron backscattered diffraction (EBSD) orientation map demonstrates the random crystallographic orientation of the individual microstructural units and apparent reduction in macro-zone size post forging, Figure 2.

\section{Mechanical Experiments}

The post heat treated pancake was sectioned into rectilinear specimen blanks and final machined into laboratory scale test pieces, Figure 3. Room temperature monotonic tensile testing was performed to BS EN ISO 6892-1:2009 [10]. Room temperature, constant amplitude load control LCF tests under a load ratio of $\mathrm{R}=0$ were conducted to BS3518-1 1993 [11], employing a 15 cycle per minute trapezoidal waveform (one second linear rise and fall ramps, one second hold at both peak and minimum load). Dwell LCF tests were essentially performed to the same standard, however, these incorporated a 120 second hold at peak load. Complete specimen rupture was the criterion employed to define fatigue failure. Fractographic and microstructural imaging was performed employing a Hitachi S4800 ultra high resolution SEM equipped with an Oxford Instruments EBSD facility at The University of Swansea or a FEI QuantaFEG 250 SEM also with Oxford instruments hardware at TIMET Witton Technical Laboratory.
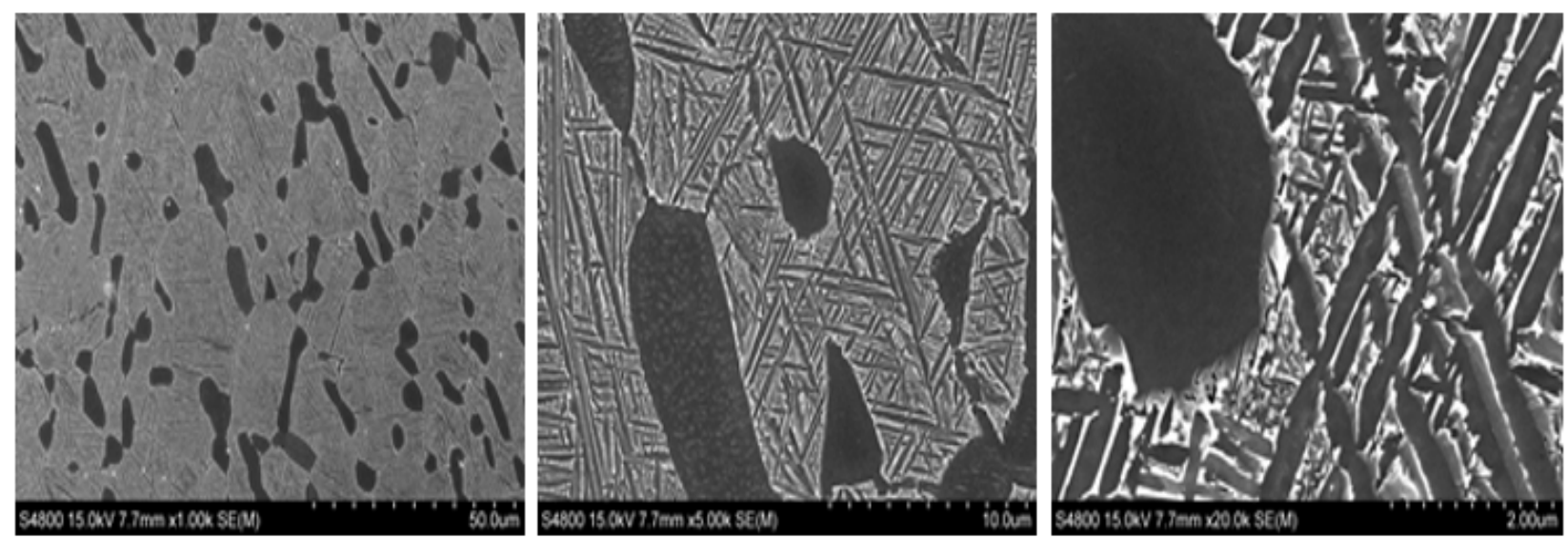

Figure 1. SE-SEM images of optimised post forged and aged microstructures at progressively higher magnifications. 

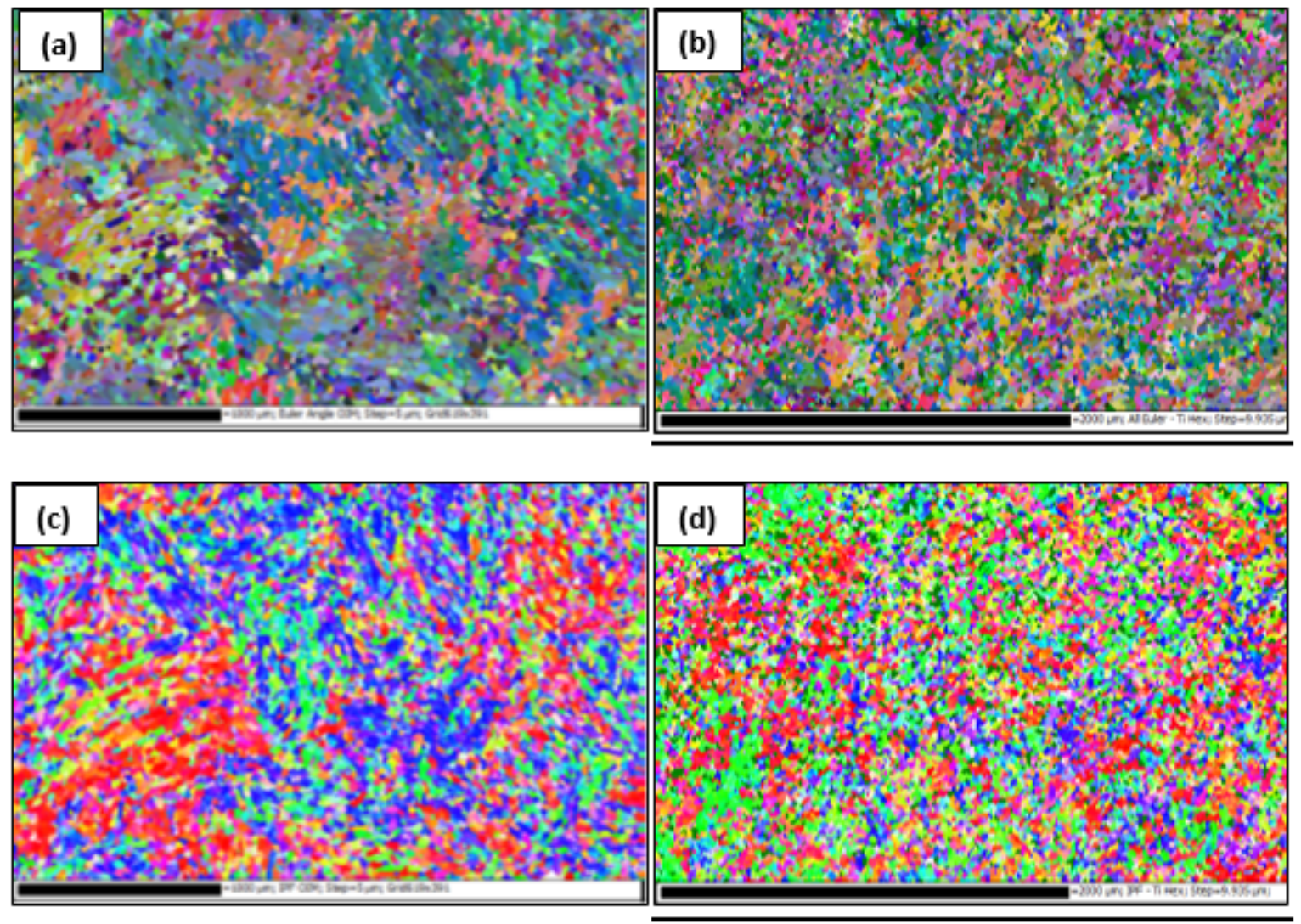

Figure 2. EBSD maps of Ti-834 microtexture using (a) Euler colouring and (c) IPF colouring (c); with Ti-575 maps from $250 \mathrm{~mm}$ diameter as-forged billet product again using (b) Euler colouring and (d) IPF colouring.

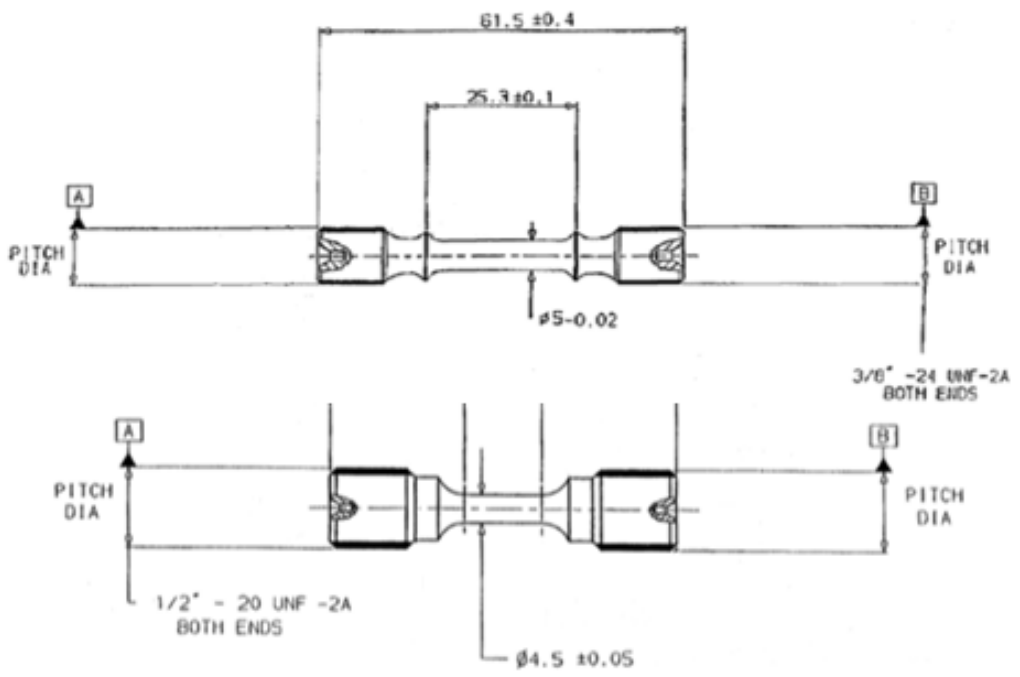

Figure 3. Plain cylindrical specimen geometries for (top) tensile and (bottom) fatigue assessment.

\section{$\underline{\text { Results }}$}

Data from three repeat tensile tests are reported in Table I as an indication of monotonic strength in the Ti-575 alloy in the force air cooled and aged condition. 
Table I. Ti-575 monotonic tension data.

\begin{tabular}{|c|c|c|c|c|}
\hline \multicolumn{5}{|c|}{ SHT Forced Air Cool and Age } \\
\hline Specimen ID & UTS (MPa) & $\mathbf{0 . 2 \% P S ~ ( M P a ) ~}$ & \% Elongation & RinA (\%) \\
\hline SHT FAC \& AGE \#1 & 1151 & 1041 & 12.0 & 46.5 \\
\hline SHT FAC \& AGE \#2 & 1149 & 1038 & 12.0 & 48.0 \\
\hline SHT FAC \& AGE \#3 & 1165 & 1049 & 12.5 & $\mathbf{1 2 . 0}$ \\
\hline Average & $\mathbf{1 1 5 5}$ & $\mathbf{1 0 4 3}$ & $\mathbf{4 6 . 0}$ \\
\hline
\end{tabular}

The LCF data measured from Ti-575 specimens are plotted in Figure 4 enabling a comparison between cyclic (15 cpm) and dwell (2 minutes) results. Across the range of life spanning 103 to 105 cycles, representative of in-service fan discs, there is clearly no sensitivity to loading waveform. Data for Ti-834 are superimposed on the same graph to emphasise the superior strength of Ti-575 under fatigue, also matching the increased static strength of the latter. But this also serves to demonstrate the dwell sensitivity of Ti-834 measured over a similar range of lives [7].

Crack initiation in the Ti-575 specimens was invariably from random, surface periphery sites, Figure 5. This follows classical theory to describe fatigue failure [12]. Inspection of cyclic and dwell specimens could not distinguish any obvious difference between the two forms of loading. Sub-surface initiation was not observed, neither was an obvious contribution from quasi-cleavage faceting.

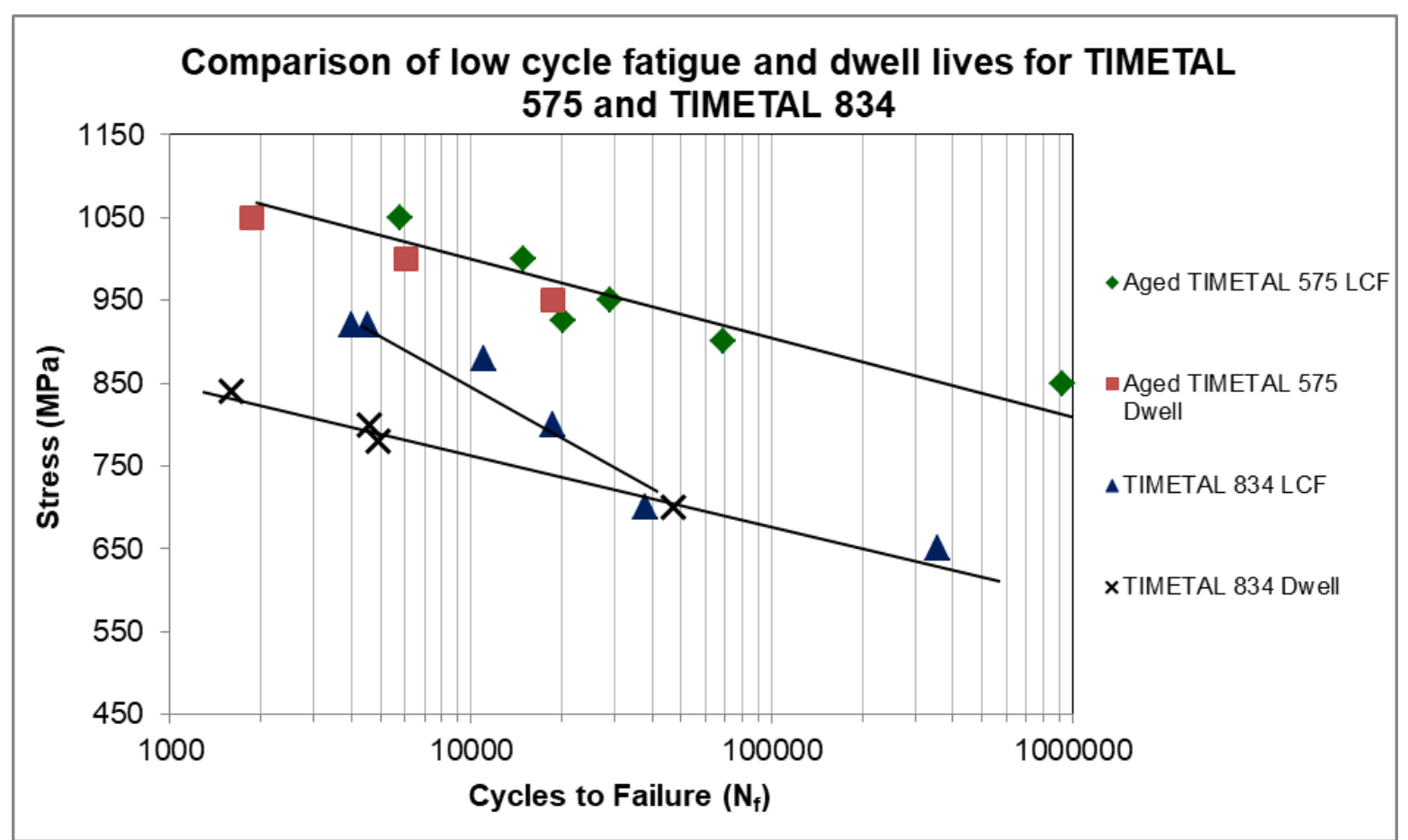

Figure 4. LCF cyclic and dwell fatigue data for Ti-575 compared to Ti-834.

\section{Discussion}

The fatigue behaviour of Ti-575, in demonstrating a clear insensitivity to dwell loading, illustrates distinct differences to the performance of Ti-834 when processed to a similar forged condition. The latter is a system considered by many to be an architype dwell alloy, where subsurface fatigue crack initiation is commonplace, associated with diffuse regions of quasi-cleavage facets formed within macro-zones of grains with preferred crystallographic orientation, Figure 6. EBSD data collected directly from the fracture surface in a previous study [13], indicate that the majority of these facets, highly flat and lustrous under optical microscopy, possessed a basal plane orientation. They are predominantly orientated perpendicular to the applied tensile test axis. It is pertinent that the fatigue data reproduced for Ti-834 in Figure 4 and associated fractography in Figure 6 relate to specimens extracted from a compressor disc forging. During the same study [7], Davies et al demonstrated that the dwell sensitivity was absent for rolled bar stock of the same alloy, free of macrozones due to more consistent and extensive hot working. This provided some of the earliest evidence for a link between thermomechanical processing, macrozone formation and dwell behaviour. Around the same period, similar conclusions were drawn by Woodfield et al. when considering another dwell sensitive alloy, namely Ti-6242 [14].

Compared to the surrounding transformed $\beta$ matrix, primary $\alpha$ grains offer a relatively strong response but are favoured for fatigue crack initiation. Even for alloys and microstructures where texture is not significant, Hines et al. demonstrated that fatigue cracks in $\alpha+\beta$ 
variants preferentially initiate from fractures within individual primary $\alpha$ grains via a process of stress redistribution [15]. It is reasonable to infer that macrozones, containing a cluster of primary $\alpha$ grains with a similar crystallographic orientation, will act as distinct, effective structural units (ESUs). In the case of Ti-834, the length scale of the ESUs can extend into millimetres, despite the typical primary $\alpha$ grain size of the order of 30 to $50 \mu \mathrm{m}$.

The EBSD orientation maps in Figure 7 straddle the boundary of a macrozone in Ti-834, demonstrating a preferred orientation for the primary $\alpha$ grains within the zone to the bottom left corner. This is best illustrated when isolating the signals collected from individual primary $\alpha$ grains. In the region immediately juxtaposed and to the top right, primary $\alpha$ grains are randomly orientated. Subject to the relative axis of loading, the zone to the top right should deform more easily, with greater variation in the inclination of basal planes amongst the microstructure. The zone to the bottom left could become highly sensitive to the regional stress redistribution, eventually forming a cluster of facets, with each associated with an individual primary $\alpha$ grain, as illustrated in Figure 6.

The lack of preferred crystal orientations in the forged Ti-575 product is seen to Ti-834 in Figure 2 . This has been achieved via a combination of chemical composition effects on the texture evolution and a carefully designed thermomechanical processing routine. Although the full details are considered proprietary, the alloy design is believed to promote broader variant selection as the $\alpha$ phase transforms from the $\beta$ phase during cooling resulting in a finer transformation product compared to alloys like Ti-6Al-4V and Ti-834. The subsequent sub-transus forging in the $\alpha+\beta$ phase field encourages breakdown of the intermediate alpha lath features that could lead to macrozones or MTR's.

Further perceived benefits of reducing the macrozone size can be seen in Figure 8. The crack, growing from left to right through this image, can be seen to traverse through the microstructure in a mixed mode fashion. The crack has both avoided selected primary $\alpha$ grains by growing around their periphery but also divides some $\alpha$ grains via a transgranular mechanism. The crack progression is also insensitive to the transformed $\beta$ phase. The result is a relatively flat, macroscopic fracture surface, near orthogonal to the cyclic tensile axis. In dwell sensitive alloys with greater microtexture it has been demonstrated that cracks may preferentially seek out the primary $\alpha$ grains in a transgranular fashion with a marked preference for growth via the basal planes [15].
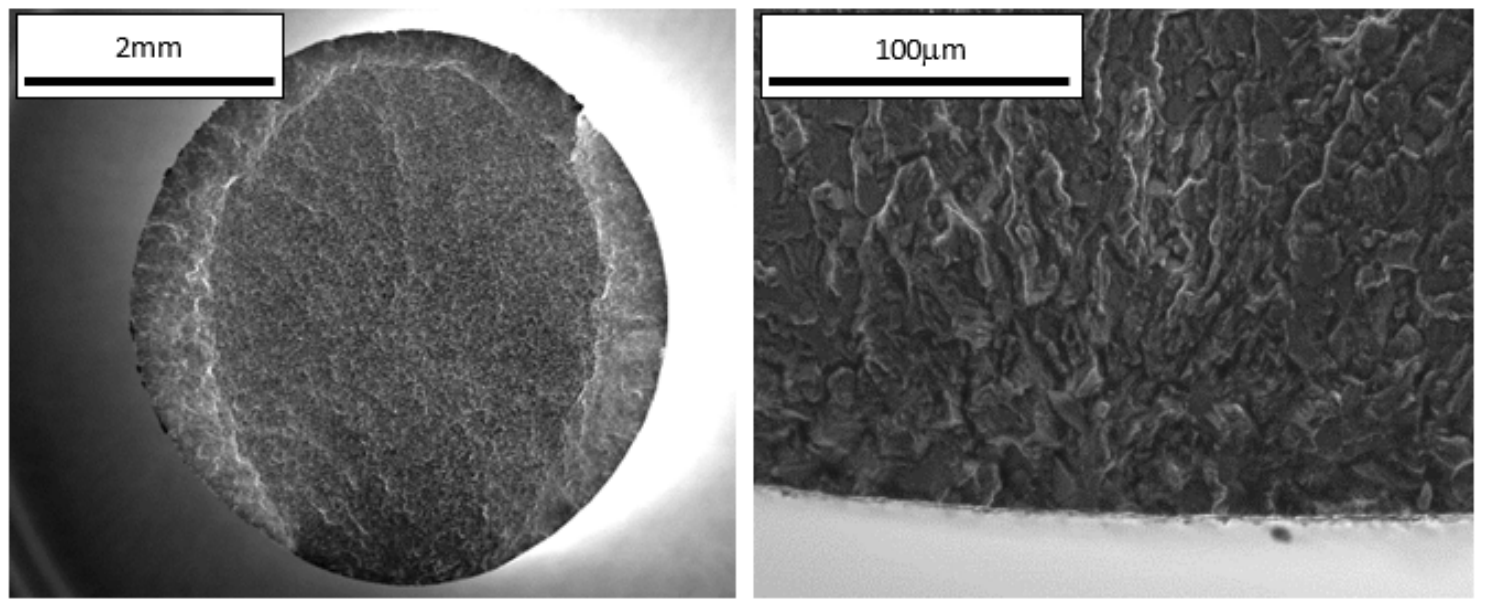

Figure 5. Typical crack initiation in a Ti-575 specimen.

\section{Conclusions}

With appropriate thermomechanical processing, the alloy Ti-575 can offer superior static strength compared to many alternative $\alpha+\beta$ titanium alloys whilst retaining good ductility. This fundamental strength is also beneficial to fatigue performance. The reduction on macrozone size appears to alleviate dwell sensitivity in Ti-575. As a candidate alloy for fan disc applications with both superior static and cyclic strength, the selection of Ti-575 could encourage the design of more compact/lighter fan discs. In turn, this could produce lighter fan system assemblies supporting higher by-pass ratios between ducted and core gas flows, thus increasing engine efficiency. 


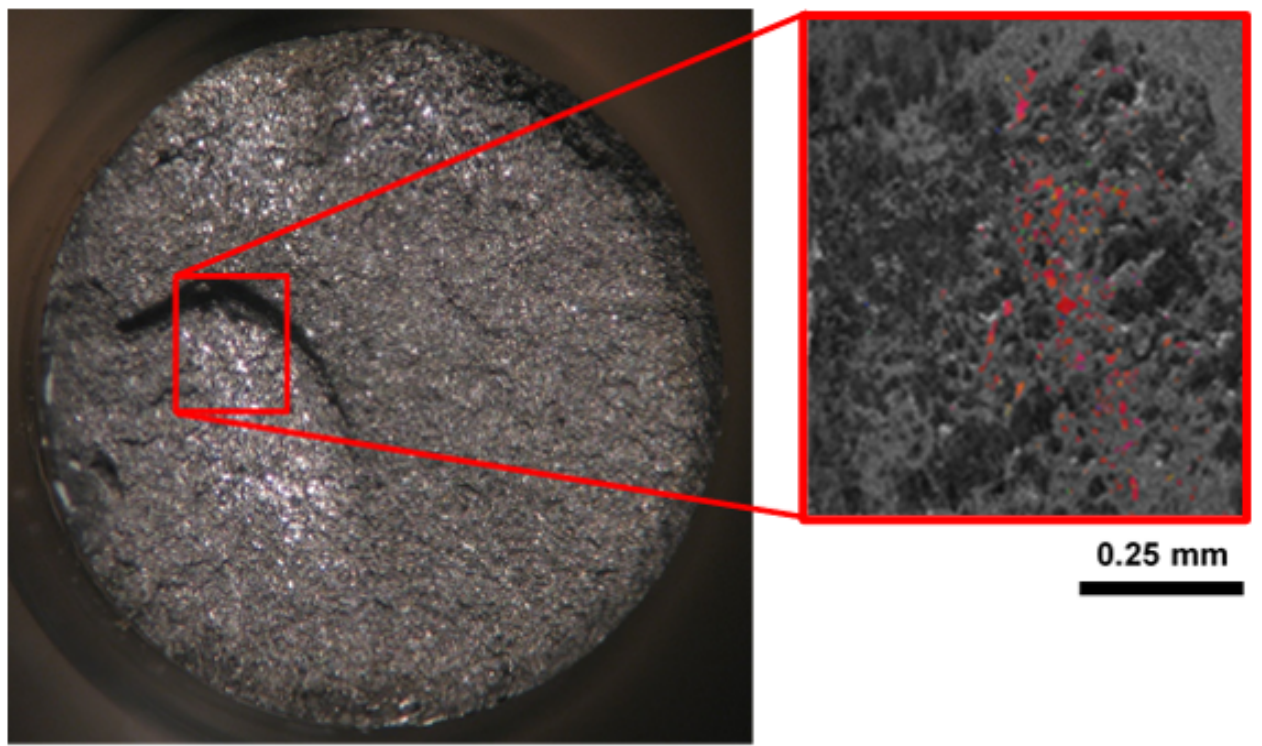

\section{$1 \mathrm{~mm}$}
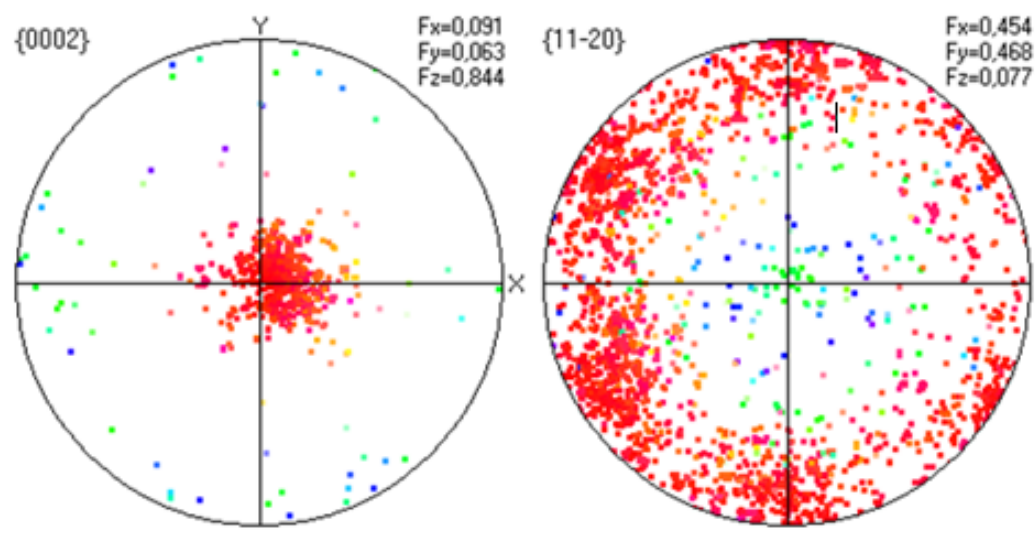

\begin{tabular}{|l|}
\hline Pole Figures \\
\hline [834_fract_tc.cpt] \\
Ti.Hex [6/mmm) \\
Complete data set \\
945 data points \\
Stereographic projection \\
Upper hemispheres \\
\hline
\end{tabular}

Figure 6. Top left: dwell fatigue failure in Ti-834; Top right: EBSD signals detected from quasi-cleavage facets; Bottom: associated pole figure data.
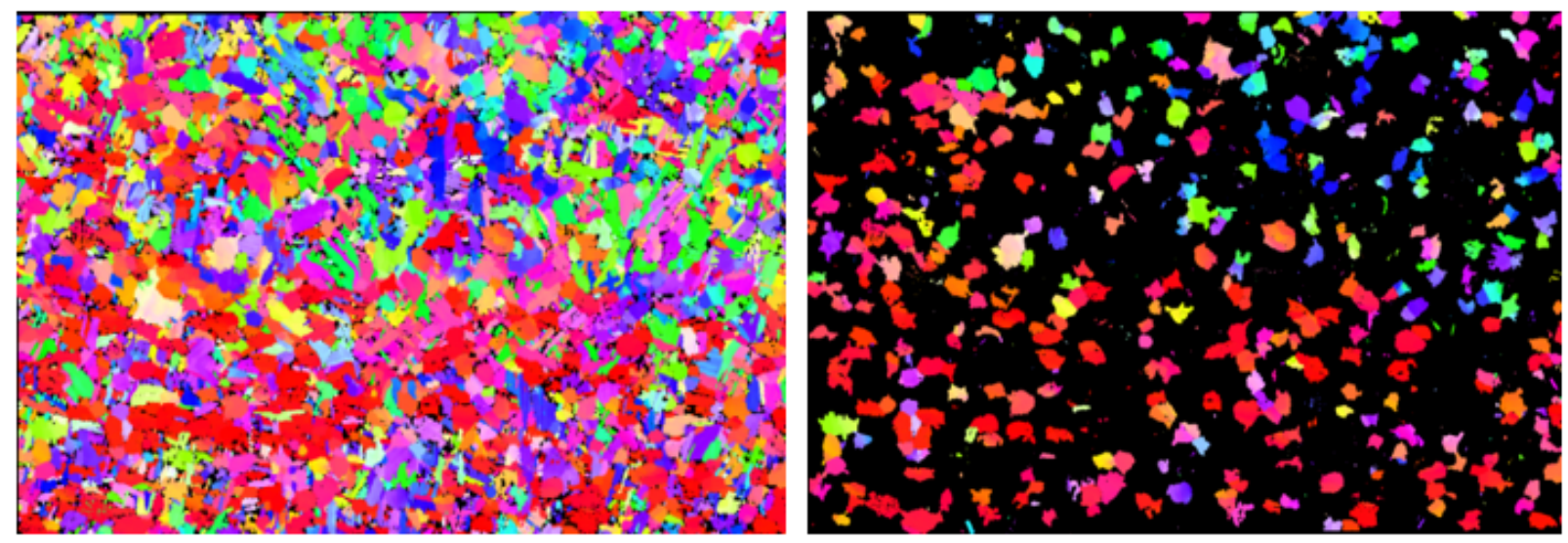

\section{$0.5 \mathrm{~mm}$}

Figure 7. Left: EBSD map from a Ti-834 microstructure; Right: same area highlighting primary $\alpha$ grains [13]. 


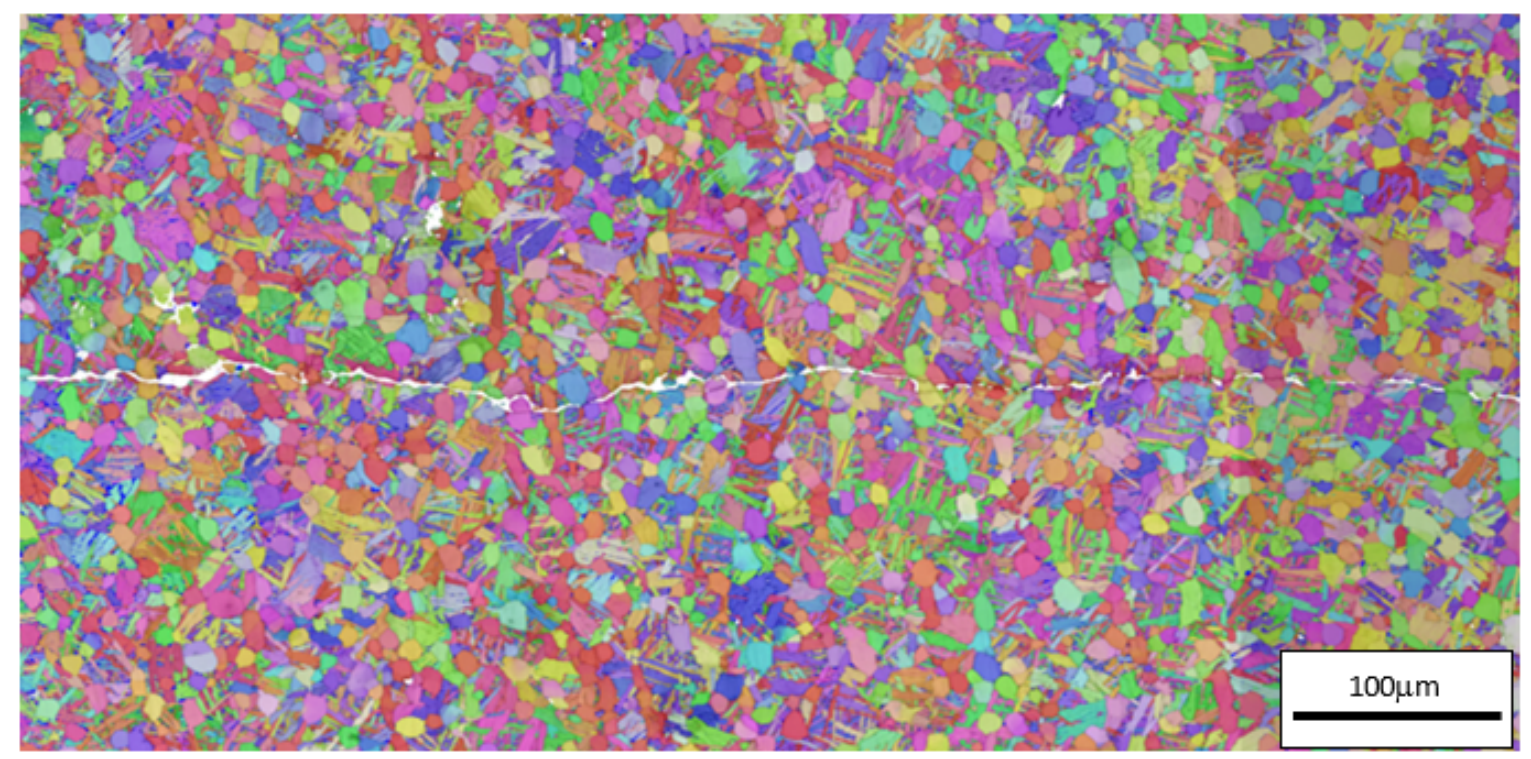

Figure 8. EBSD orientation image map (IPF) of a fatigue crack growth rate specimen.

\section{Acknowledgements}

Mechanical data, fractography and microstructural characterisation of Ti-575 were performed by Dr Jon Hewitt under an EngD study funded by the EPSRC Strategic Partnership in Structural Materials for Gas Turbines (grants EP/H500383/1 and EP/H022309/1) and sponsored by Timet UK. EBSD characterisation of Ti-834 was conducted by Dr Lionel Germain, now employed at University of Lens, France.

\section{References}

[1] P. Pugh; "The Magic of a Name: The Rolls-Royce Story", Icon Books, UK.

[2] W.J. Evans and C.R. Gostelow; Metallurgical Transactions A, 1979, Vol. 10, Issue 12, pp. 1837-1846.

[3] W.J. Evans; in: Proceedings of Third International Conference on Creep and Fracture of Engineering Materials and Structures, Institute of Materials; 1987, pp. 603-613.

[4] A.N. Stroh; Proceedings of the Royal Society London, 1954, Vol. 223, pp. 404-414, 1954.

[5] W.J. Evans, M.R. Bache;, International Journal of Fatigue, Volume 16, Issue 7, 1994, pp. 443-452.

[6] F.P.E. Dunne, D. Rugg and A. Walker;”, International Journal of Plasticity, 2007, Vol. 23, 6, pp. 1061-1083.

[7] M.R Bache, M. Cope, H.M Davies, W.J Evans and G. Harrison;, International Journal of Fatigue, Volume 19, Issue 93, June 1997, pp. 83-88.

[8] L. Germain, N. Gey, M. Humbert, P. Bocher and M. Jahazi;, Acta Materialia, 2005, Vol. 53, pp. 3535-3543.

[9] I. Weiss and S.L Semiatin, Materials Science and Engineering: A, 1999, Vol. 263, Issue 2,pp. 243-256.

[10] BS EN ISO 6892-1:2009: Metallic materials - Tensile testing - Part 1: Method of test at room temperature.

[11] BS3518-1 1993: Methods of fatigue testing. Guide to general principles

[12] S. Suresh; "Fatigue of Materials", Cambridge University Press, UK.

[13] L. Germain and M.R. Bache; in Ti-2007 Science and Technology, edited by M. Ninomi, S. Akiyama, M. Ikeda, M. Hagiwara, K. Maruyama, The Japan Institute of Metals, 2007, pp. 953-956.

[14] A.P. Woodfield, M.D. Gorman, R.R Corderman, J.F. Sutliff and B. Yamrom; in Titanium 95, Proceedings of Eighth World Conference on Titanium, Eds. Blenkinsop PA, Evans WJ, Flower HM, Institute of Materials, 1996, pp. 1116-1123.

[15] R.J. Wilson, V. Randle and W.J. Evans; Phil. Mag. A, 1997, Issue 2, pp. 471-480. 